\title{
ON THE ORIGIN OF BROAD LINE WINGS IN MOLECULAR CLOUD SPECTRA
}

\author{
Bruce G. Elmegreen \\ IBM Thomas J. Watson Research Center \\ P.O. Box 218, Yorktown Heights, N.Y. 10598 USA
}

\begin{abstract}
The broad line wings in molecular cloud spectra are proposed to result from strong magnetic waves on the periphery of dense cores and in the intercore regions where the Alfvén velocity should be larger than average. The observed line profiles are reproduced by a simple but realistic model, and the ratio of the broad to the narrow line components is found to equal approximately three, independent of cloud parameters, as long as the core/intercore contrast in the local average density is sufficiently large. Interactions between the magnetic waves should produce dense clumps in the non-linear splash regions between converging flows.
\end{abstract}

\section{Line Profiles in Magnetic Clouds with Density Gradients}

The broad line wings found in molecular cloud spectra by Blitz and Stark (1986) and Falgarone and Phillips (1990; see also Phillips, this conference) are proposed to result from non-linear magnetic wave motions in regions with gradients in the average density of gas. This result follows from a cloud model in which most of the mass is in the form of dense clumps that are clustered together into regions of higher than average clump number density. The regions with high average densities will be called cores here. Many of the cores in molecular clouds appear to be virialized. The regions with lower-than-average densities will be called intercore regions. Because the actual radiating matter is presumed to be at the uniformly high density of the clumps everywhere in the cloud, while the average density, i.e., averaged over many clumps, varies from place to place, this model agrees with the observation that the ratios of the 2-1 to the 1-0 line strengths are somewhat uniform throughout a cloud, even though the brightness temperature varies from the cores to the intercore regions (see Solomon or Falgarone, this conference).

Molecular clouds are considered to be magnetic, with average magnetic energies comparable to the gravitational and kinematic energy densities (Myers and Goodman 1988). This near equality implies that the "average" Alfvén speed in a cloud is comparable to the virial velocity. But for a cloud with a core/intercore structure, the Alfvén speed is rarely equal to its average value; it varies instead from a low value in the cores to a high value in the intercore regions. If the core/intercore average density contrast is sufficiently large, then the Alfvén speed in the intercore region can significantly exceed the virial velocity. Gas motions at such high velocities will produce excess radiation in the line wings of molecular cloud spectra, giving the impression that material is moving at greater than escape speeds, although in fact this motion should not lead to any real loss of matter from the cloud.

The molecular line profiles from magnetic clouds with average density gradients can be determined approximately by assuming that the volume emissivity is proportional to the average 
density and that the normalized line profile is Gaussian. This assumption of locally Gaussian motions is consistent with expectations from energy equipartition for an ensemble of clumps that collisionally interact (probably via non-touching magnetic linkages). Then the contribution to the line profile from the gradient region is given by

$$
I(v) \propto \int_{r_{1}}^{r_{2}} \frac{n(r)}{\sigma(r)} e^{-v^{2} / 2 \sigma(r)^{2}} d r
$$

where $r_{1}$ and $r_{2}$ span the distance over which the density and Alfvén speed vary.

Now we identify $\sigma(r)$ with the Alfvén speed at $r$ and consider that $\sigma \propto n^{-\beta}$ where $\beta$ equals 0.5 for a uniform field strength and $\beta=0.25$ for a slight field divergence along the path from the core to the intercore region. (If $\beta=0$, then there would be no broad line wings in this model.) There is a critical assumption at this step, that the magnetic wave motions contributing to $\sigma$ are non-linear; otherwise the Alfvén speed would not correspond to a material speed that can be observed by Doppler shifts in a spectrum. Non-linear waves contain mass motions both parallel and perpendicular to the mean field direction, and they also change the average density as a result of these motions. Linear waves produce only slow motions perpendicular to the field, and extremely slow motions and small density changes parallel to the field. Thus our identification of broad line wings with magnetic motions in the low density regions of clouds requires that the perturbed field strengths in these regions be a large fraction $(0.3-1)$ of the mean field strength there.

The large-scale, core/intercore density variations in a cloud are assumed to result from local equilibria between pressure gradients and self-gravitational forces. This gives $n(r) \propto r^{-\alpha}$ for $\alpha \sim$ 1 to 2 . We identify $n_{2}$ with the density in the core and $n_{1}$ with the density in the intercore region; $\sigma_{2}$ is the dispersion in the core.

For the sake of generality, we also add a contribution to the line profile from a purely Gaussian velocity distribution in the core region. This cloud core emission can be strong or weak compared to the gradient emission written in equation (1), depending on the relative emissivity of the core and intercore gas. This relative emissivity cannot be obtained from first principles, but presumably depends on the evolutionary state of the cloud if, for example, the cores become denser and more massive with time from accretion, or if the intercore region becomes denser with time from core ablation.

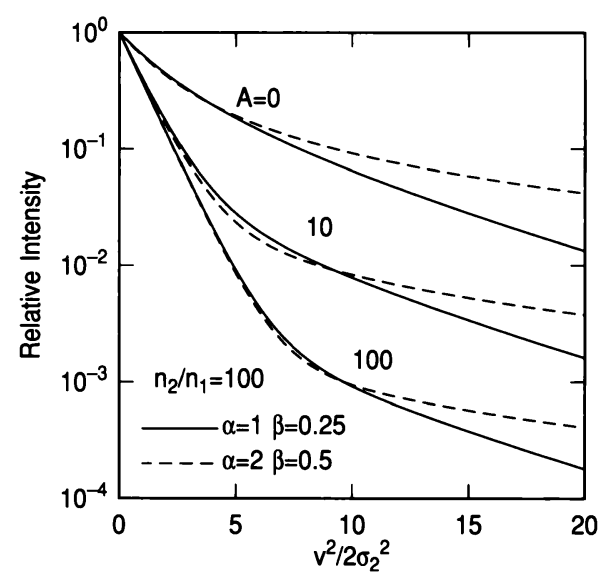

Figure 1. Sample line profiles from equation (2). 
The resultant line profile, normalized to the peak value, is

$$
\frac{I(v)}{I(0)}=\frac{\int_{C}^{1} x^{\gamma} e^{-x v^{2} / 2 \sigma_{2}^{2}} d x+A e^{-v^{2} / 2 \sigma_{2}^{2}}}{\frac{1}{\gamma+1}\left[1-C^{(\gamma+1)}\right]+A}
$$

where $\gamma=(\alpha-\alpha \beta-1) / 2 \alpha \beta$ and $C=\left(n_{1} / n_{2}\right)^{2 \beta}$. This profile is shown in Figure 1 for various $\alpha$, $\beta$, and $A$, as indicated. The coordinates for the figure are such that a Gaussian line would appear as a straight line. The excess above the extrapolated emission on the right is the line wing. These theoretical spectra resemble the observed spectra in Falgarone and Phillips (1990). Variations in the relative strength of the line core and the line wing are interpreted in terms of variations in the core and intercore masses or emissions.

The theoretical line profiles were fitted to two Gaussians for comparison with the results of Falgarone and Phillips (1990), who approximated the observed spectra in this way. The ratios of the broad to the narrow dispersions in these fits are shown in Figure 2 as functions of the core to intercore density contrast, and for various $\alpha, \beta$, and $A$. The ratio is approximately 3 , as observed for molecular clouds, for a reasonable range of parameter values as long as the core-tointercore average density contrast is not too small.

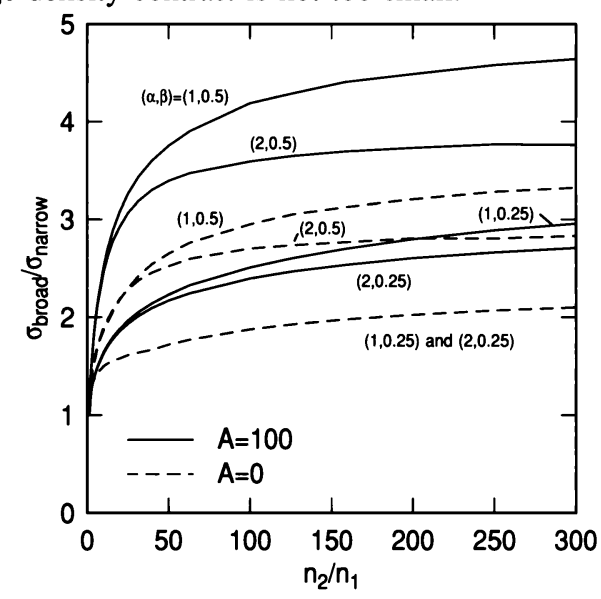

Figure 2. The ratio of Gaussian fit velocity dispersions for the broad and narrow components of the line profile generated by equation (2).

Evidently the velocity distribution functions in magnetic clouds containing strong wavelike motions and gravitationally relaxed density gradients can produce spectral line profiles from Doppler shifts that have core-wing structures similar to those observed by Falgarone and Phillips (1990).

\section{Splashy Collisions Between Non-Linear Alfvén Waves}

The previous section considered the effect of non-linear waves on the velocity distribution function in clouds with density gradients. These same waves also have the interesting property that interactions between them can produce sharp density peaks which may be visible in mo- 
lecular clouds as clumps, filaments, or sheets, depending on the wave geometry and viewing perspective. The density peaks form when the convergence of material flowing along the field lines in the non-linear wave pushes the neutrals through the ion fluid, which is attached to the field lines. The neutrals are then squeezed into a clump, filament or sheet, depending on the shape of the waves (the ion fraction continuously adjusts as this squeezing occurs, so ions should always be present in the neutral fluid.) This result follows from the non-linear equations for Alfvén waves including ion-neutral diffusion. If diffusion were not included, there would still be a density peak at the interface between the colliding wavetrains, but it would not be as large because the magnetic pressure would generally dominate the thermal pressure for typical turbulent Mach numbers in molecular clouds, i.e., for low temperatures.

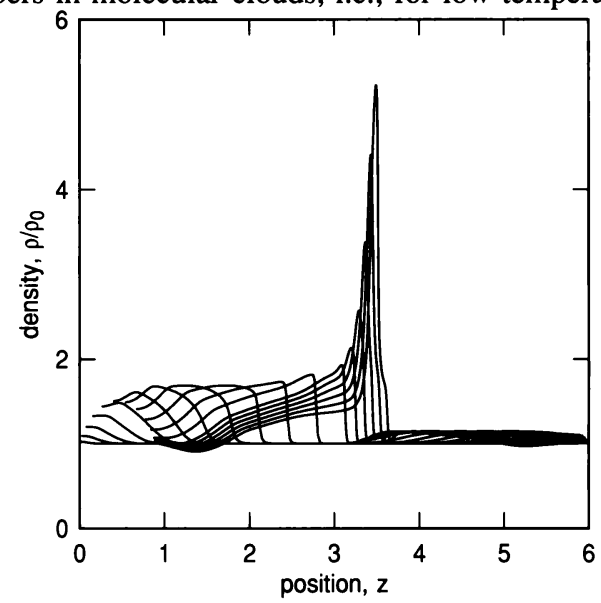

Figure 3. Density versus position of the gas in two approaching waves, with different times indicated by lines, for times 0.25 to 3 in steps of 0.25 and 3.1 to 3.5 in steps of 0.1 time units.

An example of this wave-wave interaction is shown in Figure 3, which plots the density as a function of position for different times (each line is a different time). Two waves approach each other from each side of the grid. The wave from the left is strong, driven by a transverse sinusoidal perturbation at the left hand end of the grid with a velocity equal to the unperturbed Alfvén speed. The wave from the right is weaker, driven by a transverse motion at half the Alfvén speed. Both waves contain streaming motions parallel to the field, with amplitudes equal to $\sim 0.6$ and $\sim 0.15$ of the unperturbed Alfvén speed (not shown). The plotted quantity is the gas density, which increases by only a factor of $\sim 1.5$ in the strong wave until the two waves interact. Then the density increases by at least a factor of $\sim 5$, at which point the calculation had to be stopped because it could not follow the sharp gradients.

The number of grid spacings in the calculation is 600 , and the grid points move with the parallel flow in a Lagrangian coordinate system. The computer program was tested for accuracy by comparing the speed and amplitude of the crest of a very weak wave with the Alfvén speed and expected constant amplitude from the linear theory. The program was also tested by comparing the quantities on either side of a shock front with the theoretical jump conditions for a shock. Shock fronts readily form when the waves are strong. A shock can be seen in Figure 3 when the wave from the left reaches a grid position of about 2.5. The accuracy of the computer code is estimated to be better than $3 \%$.

The formation of density peaks between colliding non-linear waves should be a common event in molecular clouds, where strong waves should abound because of virialized motions of 
the cores in the potential well of the cloud complex. The dissipation of energy that accompanies such shocks will not necessarily halt their formation because the loss of energy corresponds to a loss of pressure, which should be followed by a contraction of the whole cloud. This contraction then pumps more energy into the cloud (in the form of compressional energy, PdV), and the cloud remains in approximate virial equilibrium. The source of the energy is gravity.

Non-linear magnetic waves should be present in clouds even in the absence of stars or other direct stirring sources. They can account for the observed line profile and for some of the small scale clumpy structure.

A more complete description of this work is in Elmegreen (1990).

\section{References}

Blitz, L., and Stark, A.A. 1986, Astrophys.J.(Letters), 300, L89.

Elmegreen, B.G. 1990, Astrophys.J.(Letters)., in press.

Falgarone, E., and Phillips, T. 1990, Astrophys.J., 359, 399.

Myers, P.C., and Goodman, A.A. 1988, Astrophys.J.(Letters), 326, L27. 